\title{
THE ROLE OF THE ICT IN PROCESS MONITORING AND SYSTEM IMPACT ASSESSMENT OF RARE DISEASE COLLABORATIVE NETWORKS
}

\author{
Paolo Locatelli ${ }^{1}$, Federica Cirilli ${ }^{1}$, Eliana Panno ${ }^{1}$, Salvatore Provenzano ${ }^{2}$, Roberta Sanfilippo ${ }^{2}$ \\ and Paolo G. Casali ${ }^{2,3}$ \\ ${ }^{1}$ Fondazione Politecnico di Milano, Italy \\ ${ }^{2}$ Medical Oncology Unit 2 - Adult mesenchymal tumour and rare cancer, \\ Fondazione IRCCS Istituto Nazionale dei Tumori di Milano, Italy \\ ${ }^{3}$ Department of Oncology and Haemato-Oncology, University of Milan, Italy
}

\begin{abstract}
Rare cancers represent about $20 \%$ of the total number of cancers diagnosed in Europe, with an occurrence of less than 6 per 100.000 individuals annually. Despite the impact of each rare disease is limited, rare diseases collectively are a true challenge for public health authorities. In particular, rare cancers face specific challenges that include late/incorrect diagnosis and lack of clinical expertise.

In this context, rare cancers networks play a central role in linking national designed centres of expertise far from each other. These collaborations among clinical centres aim to improve the care quality of rare cancers in terms of patients' survival and of life quality. Moreover, the presence of a national collaborative network should induce a reduction of health migration and social costs.

To foster these networks, it is important to use ICT solutions not only to support network collaborative processes, but also to monitor the overall collaboration process and to assess the related system impact.

This paper describes the project "Italian Rare Cancer Network - Process Monitoring and System Impact Assessment" that aims to strengthen the collaborative Italian network Rete Tumori Rari (RTR) developing its quality system through an information system. The project results can be used as input for the definition of the organizational model and of the information system of the new Italian network of rare tumours (Rete Nazionale Tumori Rari) and to strengthen Italy within the European Reference Networks on Rare Adult Cancers (ERNs-EURACAN).
\end{abstract}

\section{KEYWORDS}

Rare Cancer, Collaborative Networks, Digital Health, Teleconsulting

\section{INTRODUCTION}

In social sciences, a network is defined as an association of a definite number of people connected through social relations at different levels (Héon-Klin, 2017).

In healthcare, especially for rare diseases, a network includes different clinical centres with two main roles: the providers, who are the experts in that specific rare disease, and the users, who request clinical services (e.g., teleconsultations) to the providers. Users and providers are usually far from each other.

As demonstrated by different EU funding programs (Health Program, the Connecting Europe Facility and Horizon 2020), rare diseases are still one of the priorities in EU. Indeed, there are different initiatives to support virtual networks that involve healthcare providers across Europe - European Reference Networks (ERNs) - aimed at tackling rare diseases and conditions that require highly specialised treatment and concentrated knowledge and resources.

These programs highlight two main lines of action: the exchange of information via existing European information networks on rare diseases, and the development of strategies and mechanisms for information exchange to encourage continuity of work and trans-national co-operation. To foster this information exchange, it is important to use ICT solutions (Aymé et al., 2007), not only to facilitate the collaboration 
among clinical centres and to limit the patient's travels, but also to monitor the overall process of collaboration and to assess the related system impact (Waks et al, 2013).

This paper describes an Italian project focused on the importance of the ICT in supporting diseases networks, developing a network quality system through an information system. The project results can be also used in the European Reference Networks on Rare Adult Cancers (ERNs-EURACAN), strengthening the Italian role in the network.

\section{RARE CANCER NETWORKS: SCENARIOS AND CHALLENGES}

According to the Surveillance of Rare Cancers in Europe (RARECARE) definition, rare cancers are best defined as those with an incidence $\leq 6 / 100,000$ cases per year (Gatta et al, 2011). However, when considering all of them together, rare cancers represent about $20 \%$ of the total of new cancer cases in Europe. Three main groups of rare cancers may be singled out: rare adult solid cancers $(\sim 15 \%)$, hematologic $(\sim 5 \%)$, and paediatric cancers $(\sim 1 \%)$. In Italy, professional networks are collaborating with the aim at improving the quality of care for Italian patients suffering from rare cancers. Gruppo Italiano Malattie Ematologiche dell'Adulto (GIMEMA) and Associazione Italiana di Ematologia e Oncologia Pediatrica (AIEOP) deal respectively with hematologic and paediatric cancers. Rete Tumori Rari (RTR) is a professional bottom-up network on rare adult solid cancers, operating since 1997 on a web-based platform. In 2017, Rete Nazionale Tumori Rari (RNTR) was established by the Italian government, as a service of the Italian National Health System, in order to enhance the three aforementioned professional networks and to improve the care quality of rare cancers limiting the health migration and social costs.

Moreover, in 2016, a European Joint Action on Rare Cancers (JARC) was launched to support the growth of the new European reference Networks (ERNs). The Clinical Patient Management System (CPMS) is the web-based application projected within the ERNs to be used for teleconsultations among centres at the European level.

Currently, ongoing efforts from the Italian government and the three partners of the RNTR aim at exploiting RNTR constitution to define the main requirements needed by the network organizational model and by the information system.

\section{PROJECT "ITALIAN RARE CANCER NETWORK - PROCESS MONITORING AND SYSTEM IMPACT ASSESSMENT"}

The project "Italian Rare Cancer Network - Process Monitoring and System Impact Assessment", funded by the Italian Ministry of Health, focuses on the collaborative Italian network RTR, with the aim of: (1) strengthening it and developing its quality system; (2) monitoring the collaborative process and assessing the system impact.

Indeed, the project aims at improving appropriateness through a systematic matching between individual clinical decisions and updated clinical practice guidelines. Moreover, the project aims at optimizing rare solid cancer patients' access to the most appropriate treatments by distant sharing of clinical cases. This should result in efficacy improvement, in terms of patients' survival and quality of life. The consequent reduction/rationalization of health migration should induce also social costs decreasing. Another by-product of this project is to foster clinical and translational research in rare solid cancers, gathering specific information (e.g. the number of studies related to RTR) and developing a virtual tissue bank.

The project also tests some specific functionalities on current information systems in order to support and monitor the network in its collaborative processes and to assess the related impact (e.g. in terms of healthcare migration).

The project results can be used as input for the definition of the organizational model and of the information system of the RNTR. This is a great opportunity for Italy not only to improve the quality of rare cancer treatment within the National Health System, but also at a European level, strengthening Italy within the European Reference Networks on Rare Adult Cancers (ERNs-EURACAN). 


\section{CONCLUSIONS}

The project "Italian rare care network - Process monitoring and system impact assessment" started in September 2018. The partners are currently focusing on the improvement of a clinical guidelines program, on network rules, on mechanisms by which centres may interact with each other (Hammer, 1990). The solution selected in the project to graphically representing processes is based on the Business Process Modelling Notation (Osterwalder, \& Pigneur, 2010) with specific notations to mark process instances and underline the difference between the as-is and to-be model (Weber et al., 2009).

Fondazione IRCCS Istituto Nazionale dei Tumori will evaluate how to implement additional functionalities in the RTR, focusing on its interaction with other users. Finally, the project results will be also used to define a new business model for a rare cancer network to be transferred in other Countries (Pistorio, et al., 2017). The updated results will be published in further papers.

\section{ACKNOWLEDGEMENT}

The authors would like to thank the project partners: Fondazione IRCCS Istituto Nazionale dei Tumori di Milano, Istituto Europeo di Oncologia and Fondazione Politecnico di Milano.

This work was supported by the Italian Ministry of Health (Grant No. RF-2016-02363386 to P.C.).

\section{REFERENCES}

Aymé S. et al., Networking for rare diseases: a necessity for Europe, Bundesgesundheitsblatt Journal, 50:12 (2007), 1477-1483

Gatta G. et al., Rare cancers are not so rare: the rare cancer burden in Europe, European Journal of Cancer 47:17 (2011): 2493-2511.

Hammer M. (1990). Re-engineering work: don't automate, obliterate. Harvard Business Review, 68(4):104-112.

Héon-Klin V., European Reference networks for rare diseases: what is the conceptual framework?, Orphanet Journal of Rare Diseases, (2017):12-137.

OMG (2009). Business Process Model and Notation, version 1.2.Osterwalder, A., \& Pigneur, Y. (2010). Business Model Generation (pp. 1-288). Hoboken, New Jersey: John Wiley \& Sons, Inc.

Pistorio, A., Locatelli, P., Cirilli, F., Gastaldi, L., Solvi, S., 2017. A Business Model for Digital Healthcare Environments: An Organic Approach and a Use Case for Handling Cognitive Impairment. Proceedings of the 10th International Joint Conference on Biomedical Engineering Systems and Technologies HEALTHINF, BIOSTEC, pp. 340-347.

Waks Z. et al., Analyzing the 'CareGap': assessing gaps in adherence to clinical guidelines in adult soft tissue sarcoma, Studies in Health Technology and Informatics, 186 (2013): 46-50.

Weber B, et al. (2009). Providing Integrated Lyfe Cycle Support in Process-aware Information Systems. Int'l Journal of Cooperative Information Systems (IJCIS), 18 (1), 115-165. 